\title{
Analytical model for long-distance tracer-transport in plants
}

\author{
Jonas Bühler ${ }^{\mathrm{a}}$, Gregor Huber ${ }^{\mathrm{a}, *}$, Friederike Schmid ${ }^{\mathrm{b}}$, Peter Blümler ${ }^{\mathrm{a}, \mathrm{b}}$ \\ a IBG-2: Plant Sciences, Forschungszentrum Jülich, 52425 Jülich, Germany \\ ${ }^{\mathrm{b}}$ Institute of Physics, University of Mainz, Staudingerweg 7, 55099 Mainz, Germany
}

\section{A R T I C L E I N F O}

\section{Article history:}

Received 18 August 2010

Received in revised form

2 November 2010

Accepted 2 November 2010

Available online 5 November 2010

\section{Keywords:}

Phloem

${ }^{11} \mathrm{C}$

Simulation

Data analysis

Positron emission tomography (PET)

\begin{abstract}
A B S T R A C T
Recent investigations of long-distance transport in plants using non-invasive tracer techniques such as ${ }^{11} \mathrm{C}$ radiolabeling monitored by positron emission tomography (PET) combined with magnetic resonance imaging (MRI) revealed the need of dedicated methods to allow a quantitative data analysis and comparison of such experiments. A mechanistic compartmental tracer transport model is presented, defined by a linear system of partial differential equations (PDEs). This model simplifies the complexity of axial transport and lateral exchanges in the transport pathways of plants (e.g. the phloem) by simulating transport and reversible exchange within three compartments using just a few parameters which are considered to be constant in space and time. For this system of PDEs an analytical solution in Fourier-space was found allowing a fast and numerically precise evaluation. From the steady-state behavior of the model, the system loss (steadily fixed tracer along the transport conduits) was derived as an additional parameter that can be readily interpreted in a physiological way. The presented framework allows the model to be fitted to spatio-temporal tracer profiles including error and sensitivity analysis of the estimated parameters. This is demonstrated for PET data sets obtained from radish, sugar beet and maize plants.
\end{abstract}

(c) 2010 Elsevier Ltd. All rights reserved.

\section{Introduction}

Since about a century vascular transport in higher plants is studied and analyzed, yielding in various theories and models still under debate (see e.g. Tyree and Zimmermann, 2002; Holbrook and Zwieniecki, 2005; Lacointe and Minchin, 2008; De Schepper and Steppe, 2010). Because of the delicate structure of the transport tissues, non-invasive techniques are needed to study the underlying mechanisms in intact plants. Very powerful approaches in that respect are tracer studies using radioisotopes (Vose, 1980) or magnetic resonance imaging (MRI) (Ishida et al., 2000; Köckenberger, 2001). With both modalities, spatio-temporal data sets can be obtained by monitoring a labeled item (either radioactive tracer or excited nuclear spins) inside the plant. Although MRI is capable to directly measure velocities of ${ }^{1} \mathrm{H}_{2} \mathrm{O}$ in the two major long-distance tissues of plants, xylem and phloem (Windt et al., 2006), its spatial resolution does not reach cellular and hardly vascular dimensions. The same restriction holds for the spatial detection of radioactive tracers, where either autoradiographic projections integrate over the third dimension or as in the case of positron emission tomography (PET) the range of the positrons before annihilating limits spatial resolution to approximately $1.4 \mathrm{~mm}$ (Phelps et al., 1975). Hence, the pixels (2D) or voxels (3D) integrate over several tissues and the related local transport processes. Consequently, the acquired data incorporates information of different processes (e.g. flow, diffusion, exchange, storage, reactions, etc.), which

*Correspondence to: Forschungszentrum Jülich, Leo-Brandt-Strasse, 52428 Jülich, Germany. Tel.: +49246161 1503; fax: +492461612492.

E-mail address: g.huber@fz-juelich.de (G. Huber). typically cannot be disentangled experimentally (Jahnke et al., 2009). Therefore, the extraction of the averaged transport parameters needs a simplified model of the underlying processes in order to relate them to predictions from physiological models or experimental hypotheses.

The aim of this work is not to add another mechanistic transport model or theory to the pool of existing ones (see e.g. Table 1 in Thompson and Holbrook, 2003), but to suggest a simplified physical description of the spatio-temporal data from MRI or PET measurements. Existing models of mass transport in plants (Christy and Ferrier, 1973; Goeschl and Magnuson, 1986; Thompson and Holbrook, 2003; Hölttä et al., 2006; Pickard and Abraham-Shrauner, 2009) are typically considering many physiological parameters and hence are too complex for this purpose, but could of course be very useful to physiologically interpret the results of our data analysis.

On the other hand, we want to refrain from a solely data driven approach like time series analysis (e.g. Minchin and Troughton, 1980) for the following reasons: (a) it completely disregards physical parameters of which at least the boundaries or ranges are typically known, (b) the error analysis of autoregressive polynomial fits is complex, (c) de-trending the data from the rapid radioactive decay of short lived isotopes is non-trivial and finally (d) the use of system identification theory is problematic in cases where input and output are very similar as it is usually the case for homogeneous spatial sections along the vascular transport pathway of plants. Due to these reasons, we request the following properties for a suitable model. It should

(a) represent transport of a non-specific tracer like ${ }^{11} \mathrm{C}$ for PET or a stable contrast agent like ${ }^{2} \mathrm{H}_{2} \mathrm{O}$ for MRI within vascular conduits 
of higher plants as well as lateral exchange with the direct vicinity of the conduits;

(b) be physical and as simple as possible to represent the data obtained with very different techniques and to be applicable to (m)any plants;

(c) be a mechanistic transport model without any scaling parameters (sizes) or anatomical details. In a separate approach, further biological theories and physiological models can then explain the model parameters and their responses to e.g. environmental changes;

(d) include only a minimum number of fit parameters, too many could make the model too specific and the fitting result might become ambiguous;

(e) possibly be solvable analytically in order to speed up the fitting procedure;

(f) contain measurable transport constants like diffusion coefficients or velocities to enable reasonable physical constraints for a fitting procedure;

(g) allow for arbitrary input (initial) tracer distributions in order to cover all kinds of experimental situations;

(h) allow to represent spatio-temporal data, i.e. time series of the spatial changes of the amount of tracer along the transport trajectory.

The model presented here is based on previously published models for tracer transport (Horwitz, 1958; Tyree, 1975) and is similar to models known from other disciplines (Krantz, 2007), e.g. chromatography (van Deemter et al., 1956) or dialysis (Legallais et al., 2000) but was adapted and extended to meet the specific requirements mentioned above.

\section{Three compartment tracer transport model}

\subsection{Model description}

The basic scenario for our model is illustrated in Fig. 1, where the tissue of the plant that participates in transport is divided into three compartments:

1. The vascular conduit (xylem or phloem vessel) of undefined size with coherent transport (flow, drift and convection) with one effective velocity, $v$, and an incoherent diffusion with coefficient $D_{1}$.

2. A parenchyma of unspecified width and sort. Exchange from compartment 1 to compartment 2 is described by a rate $a_{12}$, and

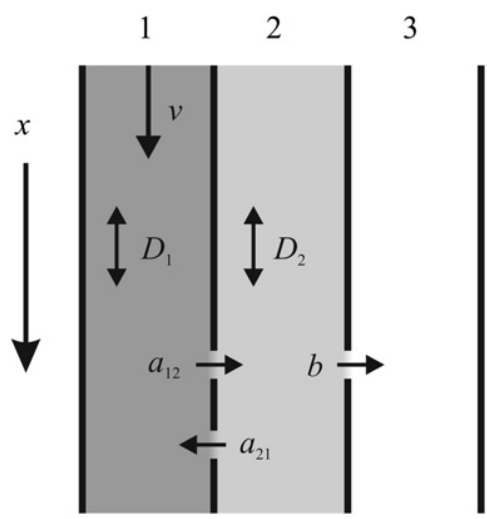

Fig. 1. Schematic representation of the mechanistic transport model with 3 compartments. The system is observed along an arbitrary spatial transport trajectory $x$ with respect to time $t$. The observed signal is an integral of the three compartments. $v$ is the flow velocity in compartment $1, a_{12}, a_{21}$ and $b$ represent the exchange rates between the compartments and $D_{1,2}$ are the diffusion coefficients for the first and second compartment. in the opposite direction by a rate $a_{21}$. There is no coherent transport but only diffusion with a coefficient $D_{2}$.

3. Finally, the tracer can become completely immobilized in the third compartment. Once adsorbed from the second compartment by a rate $b$, it stays there (e.g. metabolized ${ }^{11} \mathrm{C}$ in form of cellulose).

Radioactive decay is represented by a decay rate, $\lambda=\ln 2 / t_{1 / 2}$, with $t_{1 / 2}$ as the half-life of the used isotope (for ${ }^{11} \mathrm{C}: t_{1 / 2}=20.4 \mathrm{~min}$ and $\lambda=5.67 \times 10^{-4} \mathrm{~s}^{-1}$; for ${ }^{18} \mathrm{~F}: t_{1 / 2}=110 \mathrm{~min}$ and $\lambda=1.05 \times 10^{-4} \mathrm{~s}^{-1}$; and for stable isotopes: $t_{1 / 2}=\infty$ and $\lambda=0 \mathrm{~s}^{-1}$ ). Hence, this model consists of transport and exchange parameters only and contains no details on spatial scales or anatomy. We avoided an additional fourth compartment representing a vascular conduit with reverse flow compared to compartment 1 because in typical ${ }^{11} \mathrm{C}$ tracer experiments exchange of tracer between phloem and xylem has no significant impact on the data (Thorpe et al., 1998). The main extensions of our model compared to previous two-compartment models (Horwitz, 1958; Tyree, 1975) are the third compartment and diffusion terms for the first and second compartment.

According to Fig. 1 the change of the density in space $(x)$ and time $(t), \rho_{i}(x, t)$ (arbitrary units), of the observed signal carrier in the three compartments $i=1,2,3$ can be completely described by Fokker-Planck equations (Risken, 1989) including exchange terms

$$
\begin{aligned}
& \text { 1: } \frac{\partial \rho_{1}(x, t)}{\partial t}=-v \frac{\partial \rho_{1}(x, t)}{\partial x}+D_{1} \frac{\partial^{2} \rho_{1}(x, t)}{\partial x^{2}}-\left(a_{12}+\lambda\right) \rho_{1}(x, t)+a_{21} \rho_{2}(x, t) \\
& \text { 2: } \frac{\partial \rho_{2}(x, t)}{\partial t}=D_{2}^{\frac{\partial^{2} \rho_{2}(x, t)}{\partial x^{2}}+a_{12} \rho_{1}(x, t)-\left(a_{21}+b+\lambda\right) \rho_{2}(x, t)} \\
& \text { 3: } \frac{\partial \rho_{3}(x, t)}{\partial t}=\underbrace{D_{\text {(flow) }}}_{\text {coherent motion }} \underbrace{}_{\text {incoherent motion }} \underbrace{b \rho_{2}(x, t)-\lambda \rho_{3}(x, t)}_{\text {exchange, decay and adsorption }}
\end{aligned}
$$

where $v$ is the effective velocity in compartment 1 . We assume a single effective velocity, $v$, (corresponding to "plug flow" with typical velocities around $10^{-5} \mathrm{~m} / \mathrm{s}$ for phloem and $10^{-3} \mathrm{~m} / \mathrm{s}$ for xylem) because this allows to neglect sizes and shapes of the compartment as well as additional obstacles (e.g. sieve plates). This coarse simplification of a probably laminar velocity distribution is justified by our requirement to keep the model as simple as possible. We also assume constant and concentration independent diffusion coefficients. The exchange parameters, $a_{12}, a_{21}$ and $b$ are rates like the radioactive decay rate $\lambda$ and have units $1 / \mathrm{s}$. The observed quantity however is the sum over all three compartments, because the applied methods cannot resolve the proposed compartments with sufficient spatial resolution, hence

$\rho=\rho_{1}+\rho_{2}+\rho_{3}$.

\subsection{Analytical solution}

We first consider a situation where tracer enters compartment 1 only at some given time $t=0$. Hence the tracer distribution at that time is assumed as $\rho_{1}(x, t=0)=\rho_{0}(x)$ and $\rho_{2,3}(x, t=0) \equiv 0$. The system of partial differential equations (1) then has the following solution in reciprocal space $k$ (wave number), after or before Fourier-transformation

$\hat{\rho}(k, t)=\hat{\rho}_{0}(k) e^{-\lambda t}\left\{e^{-\beta t}\left[\cosh (\delta t)(1-\alpha)+\sinh (\delta t) \frac{a_{12}-\alpha \beta-\gamma}{\delta}\right]+\alpha\right\}$

with the definitions

$\alpha=\frac{a_{12} b}{\beta^{2}-\delta^{2}}$

$\beta=\frac{1}{2}\left(a_{12}+a_{21}+b+i k v+k^{2}\left(D_{1}+D_{2}\right)\right)$ 
$\gamma=\frac{1}{2}\left(a_{12}-a_{21}-b+i k v+k^{2}\left(D_{1}-D_{2}\right)\right)$

$\delta=\sqrt{\gamma^{2}+a_{12} a_{21}}$

The derivation of the solution can be found in Appendix A. Eq. (3) represents the total density $\hat{\rho}(k, t)$, at time $t$ in Fourier-space as a product of the expression on the right side with the initial density $\hat{\rho}_{0}(k)$, hence as a convolution of the two in real space, as to be expected in a linear system. Therefore, an expression in $k$-space not only simplifies the equation but can become a real advantage, because some of the data are already originally acquired in reciprocal space (e.g. MRI data) or can be readily numerically transformed. Another obvious result is that all of the initial signal has to decay with $\exp (-\lambda t)$.

If the initial condition cannot be obtained in another way, in the case of pulse-labeling experiments it is convenient to assume a simple Gaussian distribution $\rho_{0}(x) \sim \exp \left(-\left(x-x_{0}\right)^{2} / 2 \sigma^{2}\right)$, which corresponds in Fourier-space to

$\hat{\rho}_{0}(k) \sim \exp \left(-\frac{1}{2} \sigma^{2} k^{2}-i x_{0} k\right)$

Here $\sigma$ is the width of the distribution and $x_{0}$ is the shift into the negative spatial direction. This initial condition is not meant to mimic any realistic labeling process but merely provides an input function roughly consistent with the nature of the experiment. A performance comparison of the analytical solution with a standard PDE solver can be found in Appendix C.

\subsection{General behavior of the model}

In a special case where all model parameters are zero except for velocity and exchange to compartment $2\left(v>0, a_{12} \geq 0\right)$, the solution of Eq. (1) reduces to a simple expression in real space, see Appendix B. In a case where more parameters are different from zero, the analytical expression (3) no longer provides an intuitive insight into the general behavior of the model. In order to illustrate this behavior and the influence of the model parameters the tracer distribution in each compartment is exemplified in Fig. 2.

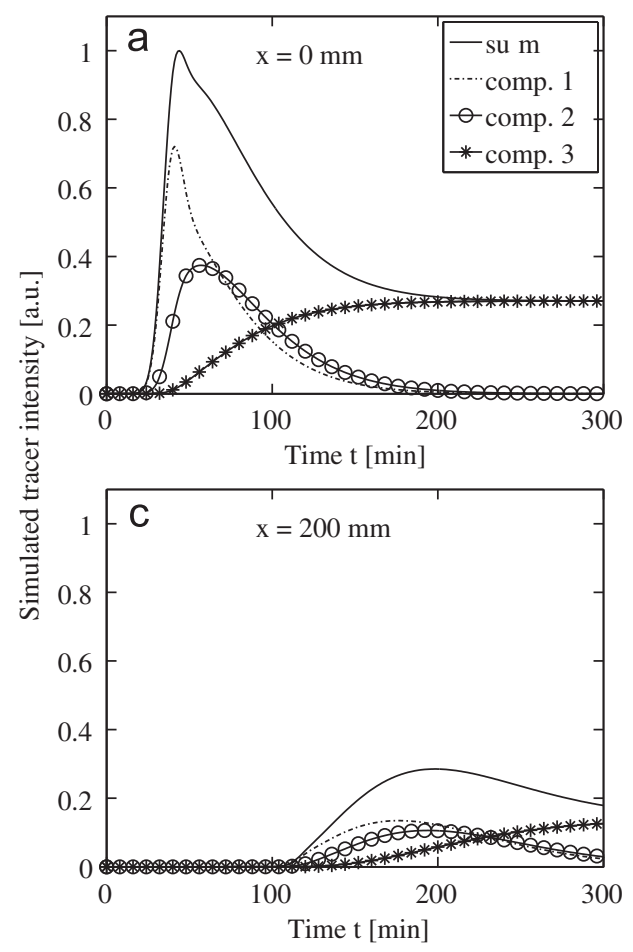

Parameter $a_{12}$ determines exchange of material to compartment 2 where it stays until it exchanges back to compartment 1 or moves to compartment 3 . The exchange rate $a_{21}$ brings matter back into the stream of compartment 1 and causes the tail of the data for compartment 1 at higher values of $x$ and $t$. Storage in compartment 3 is described by parameter $b$. With non-zero $b$ material stays mostly at low $x$-values as a constant (see Fig. 2a), depriving the system so much that an exponential decline is observed also in the spatial domain. The width of the time series for compartment 2 is mainly determined by the sum of $a_{21}$ and $b$.

For the simulated tracer distribution shown in Fig. 2, the decay constant was set to zero $(\lambda=0)$ in order to allow a visualization of the tracer distribution for high values of $t$ and $x$. Here and in all following calculations, the diffusion constants are set to zero $\left(D_{1}=D_{2}=0 \mathrm{~m}^{2} / \mathrm{s}\right)$ for the following reason: the diffusion coefficient of water is known to be in the order of $2 \mathrm{e}-9 \mathrm{~m}^{2} / \mathrm{s}$. We assume the self-diffusion of the photoassimilates within the phloem to be even slower and negligible in comparison to changes of the tracer concentration caused by transport- and exchange processes.

\subsection{Model behavior in steady state}

Next we consider a scenario where the system is fed by a constant flow $j_{0}$ (dimensions of $\rho$ times $[\mathrm{m} / \mathrm{s}]$ ) of tracer at the position $x_{0}$, resulting at $t \rightarrow \infty$ in a steady state in compartments 1 and 2, whereas compartment 3 accumulates tracer. The fraction of tracer fixed in compartment 3 along a certain spatial distance $x_{2}-x_{1}$ is a physiologically meaningful parameter and often called 'loss' or 'leakage' (Minchin and Thorpe, 2003). Since the quantity of interest here is the total amount of immobilized tracer and not the amount of tracer that is still radioactive, we set the decay rate to zero in the following $(\lambda=0)$. The PDEs from Eq. (1) then simplify to

$$
\begin{aligned}
& 0=-v \frac{\partial \rho_{1}(x, t)}{\partial x}-a_{12} \rho_{1}(x, t)+a_{21} \rho_{2}(x, t) \\
& 0=a_{12} \rho_{1}(x, t)-\left(a_{21}+b\right) \rho_{2}(x, t)
\end{aligned}
$$

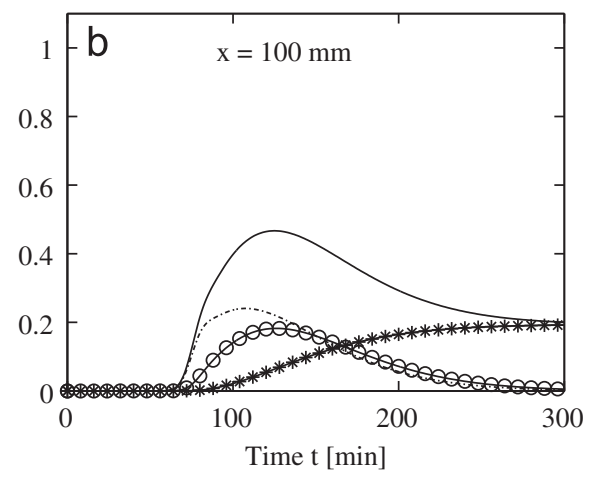

Parameters:

$\mathrm{v}=2.5 \mathrm{~mm} / \mathrm{min}$

$\mathrm{a}_{12}=0.05 \mathrm{~min}^{-1}$

$\mathrm{a}_{21}=0.05 \mathrm{~min}^{-1}$

$\mathrm{b}=0.01 \mathrm{~min}^{-1}$

$\sigma=15 \mathrm{~mm}$

$\mathrm{x}_{0}=100 \mathrm{~mm}$

$\mathrm{A}_{0}=1.0$ [a.u.]

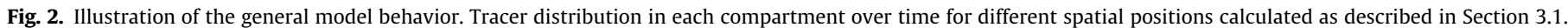
Diffusion and decay have not been regarded; $D_{1}=D_{2}=0, \lambda=0$. (a) $x=0 \mathrm{~mm}$, (b) $x=100 \mathrm{~mm}$, (c) $x=200 \mathrm{~mm}$. 
$\frac{\partial \rho_{3}(x, t)}{\partial t}=b \rho_{2}(x, t)$

with the boundary condition $\rho_{1}\left(x_{0}\right)=j_{0} / v$. This can directly be solved to $\rho_{3}$

$\rho_{3}(x, t)=j_{0} \vartheta \exp \left(-\vartheta\left(x-x_{0}\right)\right) t$

with the substitution

$\vartheta=\frac{a_{12} b}{v\left(a_{21}+b\right)}$

The loss can be calculated as the ratio of tracer fixed in the region of interest to the tracer entering this region:

Loss $=\frac{\int_{x_{1}}^{x_{2}} \rho_{3} d x}{\int_{x_{0}}^{\infty} \rho_{3} d x-\int_{x_{0}}^{x_{1}} \rho_{3} d x}=1-\exp \left(-\vartheta\left(x_{2}-x_{1}\right)\right)$

As this parameter only depends on the transport and exchange properties of the model and is largely independent of the input function, it is especially useful when comparing results from different experimental scenarios.

\section{Analyzing experimental data}

Numerical evaluation, fitting and error analysis was computed in MATLAB R2010a (MathWorks, Inc.) using mainly home-built procedures, which can be requested from the authors.

\subsection{Fitting procedure}

Fitting means minimizing the merit function (Press et al., 2007), being the difference of the simulated data to the experimental data, by variation of the model parameters. In a first step, the experimental data are read in and the fitting procedure is started by guessing good start parameters (typically done by visual inspection). The evaluation of the analytical equation (3) is implemented as follows:

(a) The time points of interest $\left[t_{\min } \ldots t_{\max }\right]$ are known from the experiment. The spatial grid, $d x$, is defined with regard to the desired numerical accuracy and the available computing time (see Fig. 6 in Appendix C).

(b) The maximal extension in $k$-space is calculated as $k_{\max }=\pi / d x$.

(c) The velocity, $v$, results in a guess of the maximal spatial extension $x_{\max }=v\left(t_{\max }-t_{\min }\right)+x_{0}$. Real space then stretches from $-x_{\max }$ to $+x_{\max }$, hence having $n=2 x_{\max } / d x$ entries. The gridding of $k$-space, $d k$, is then simply defined as $d k=$ $2 k_{\text {max }} / n=\pi / x_{\text {max }}$. (d) If the initial density function $\hat{\rho}_{0}(k)$ cannot be quantified by an already existing expression of Eq. (3) another function has to be assumed. Typically, in the case of pulse-labeling experiments we use the Gaussian from Eq. (4) with width $\sigma$ and shift $x_{0}$, both of which are treated as fitting parameters as well.

(e) Eq. (3) is then numerically evaluated on this $k-t$ grid and discretely Fourier-transformed along the $k$-direction only.

(f) Because $\rho(x, t)$ denotes a concentration of matter, the result of step (e) must be real and positive. However, caution must be paid to the fact that numerical errors and various implementations of the discrete FFT can cause a non-zero imaginary part. Affirming that the origin of an imaginary result is purely due to finite numerical accuracies, it can simply be ignored by taking either the real or complex magnitude value.

(g) $\rho(x, t)$ is interpolated to the spatial and temporal region of interest and multiplied with a scaling factor ensuring the simulation to be in the right order of magnitude. This scaling factor is also fitted later on.

Fig. 3 shows a result for $\rho(x, t)$ which is typical as being similar to the experimental data analyzed in the following.

In a next step the best fit to the data is searched by a standard non-linear least-squares method (Levenberg, 1944; Marquardt, 1963; Press et al., 2007) utilizing a damping strategy as described in (Nielsen, 1999). The necessary partial derivates of the meritfunction are approximated numerically using the first-order central differential quotient. Additionally, the full parameter space is restricted to the positive subspace only, because all parameters in Eq. (1) are greater or equal zero. Regarding the velocity, $v$, data preparation has to be performed in such a fashion that $v \geq 0$. In order to force the fitting routine to search for a minimum in this parameter subset only, it operates on the logarithm of the parameter space.

\subsection{Error analysis}

Due to the calculation of the fitting function in a Fouriertransformed domain the standard procedure of error estimation (using co-variances) in Levenberg-Marquardt fits could not be implemented in a straight-forward way. Therefore, a statistical (Monte-Carlo simulation, sampling bootstrapping) approach was used to determine confidence intervals of all parameters (Press et al., 2007). This approach randomly replaces some data points with others using an appropriate distribution to generate modified pseudo-experimental data sets, each of which is then fitted again. This procedure is repeated as often as possible and the distribution
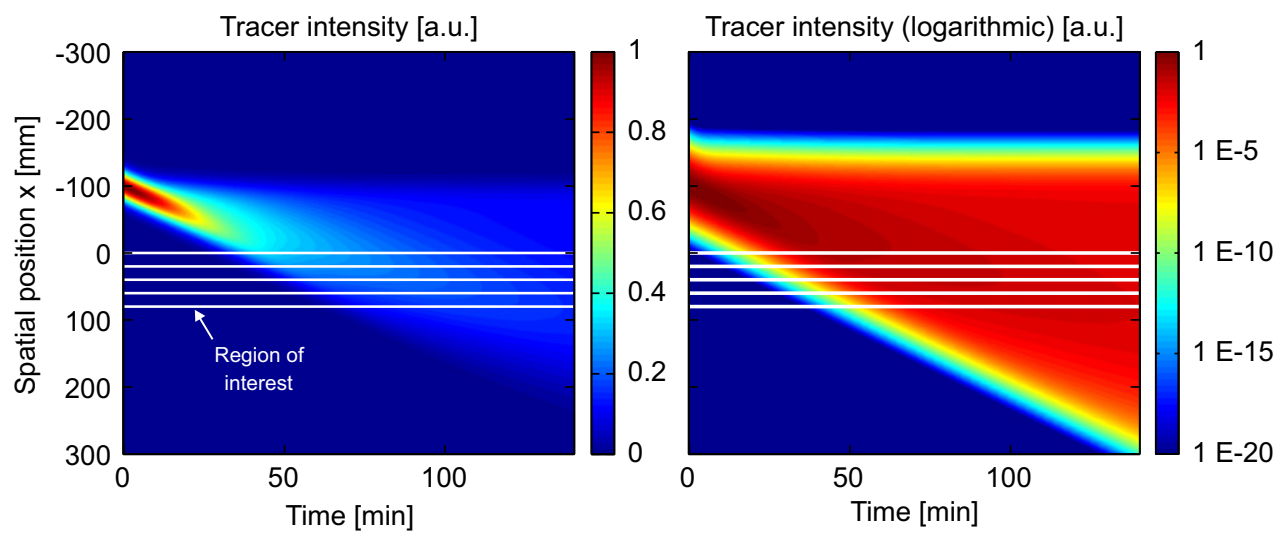

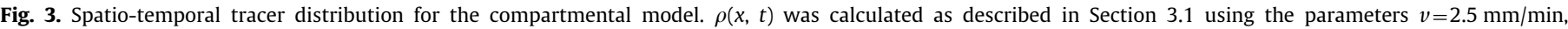

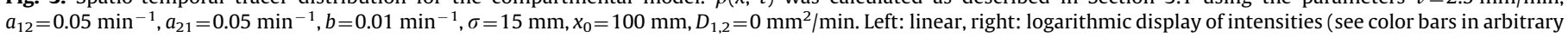

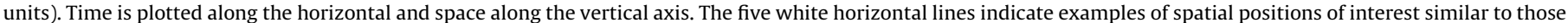
used in the later analysis of experimental data. 
of the resulting fitted parameter sets is assumed to be the error distribution of the according parameter. In this work, we used a uniform distribution for generating new data sets with a bootstrap sampling rate of 400 and analyzed the resulting errors as being normally distributed. For more details see (Press et al., 2007).

\subsection{Sensitivity analysis}

The sensitivity of the model regarding changes of the parameters can already give a hint how fast each parameter can be estimated when the model is fitted to artificial or experimental data. In order to determine the sensitivity each parameter was varied stepwise from $-50 \%$ to $50 \%$ in relation to the parameter set from Fig. 3. The deviance $\chi^{2}$ is the sum over the squared deviations from the reference data. In this range of the parameter space and considering the same spatial and temporal positions as used in Section 2.3, Fig. 4a clearly shows the dominant influence of the velocity $v$ and the exchange rate $a_{12}$. For all parameters $\chi^{2}$ shows an unsymmetrical development for higher values $(\Delta p>25 \%)$ which is due to the strongly non-linear nature of the solution Eq. (3).

By adding noise to the same reference data and fitting the resulting curves as described in Section 3.1, the confidence intervals of each model parameter can be calculated according to Section 3.2. The result, as shown in Fig. $4 \mathrm{~b}$, already indicates the uncertainty to be expected from a certain level of noise in experimental data. The velocity $v$ shows the smallest uncertainty in accordance with the results in Fig. 4a. For the other parameters there is no such direct relationship between impact on the model behavior and confidence interval since their uncertainty strongly depends on the respective spatial and temporal grid.

\subsection{Example: PET data of ${ }^{11} \mathrm{C}$ transport into three different plant roots}

The data presented in Fig. 5 were obtained from spatiotemporally resolved PET data on three different root types (sugar beet, radish and maize) and their acquisition is explained elsewhere (Jahnke et al., 2009). For the data from the sugar beet and maize root experiments the three-dimensional spatial data were integrated over two dimensions and a certain width in the third dimension. This third dimension is roughly the gravitational axis along which transport in plants occurs. Hence, the result of this crude data simplification is somewhat mimicking the traditional approach to place integrating detectors along the transport pathway. In order to exploit the full wealth of information in the 4D PET/ MRI data much more sophisticated tools need to be developed in the future. A first approach in this direction was implemented for the data from the radish experiment where it is obvious that the experimental transport pathway is curved. Here cylindrical masks were distributed along the major transport pathway (see Fig. 5c). The time profiles at the centers of the masks were extracted by integrating the data over these masks.

The data displayed in the following are therefore projections of the transported ${ }^{11} \mathrm{C}$ tracers on a one-dimensional pathway through the root system. These time series were analyzed as described in Sections 3.1 and 3.2. The resulting fitted parameters and the $90 \%$ confidence intervals are shown in Table 1.

A similar fit was done for a modified model with a fixed ratio between the parameters $a_{21}$ and $a_{12}: a_{21}=h a_{12}$, where $h$ is a constant. The idea behind this modification is to simplify the model such that the number of fitting parameters is reduced without affecting the general functionality of the model too much. Table 2 shows the fitting results for all three data sets using different values of $h$. Firstly, $h$ was set to the ideal ratio of $a_{12}$ and $a_{21}$ resulting from the fit in Table 1 . As expected, the ideal ratio delivers the original fitting values from Table 1 but with considerably smaller confidence intervals. Using arbitrary values for $h(0.5,1.0$ and 2.0$)$, the fitted parameters somehow differ from Table 1 but the confidence intervals also decreased significantly.

\section{Discussion}

Our focus on rigorous parameter estimation is motivated by the goal of providing a method for quantitative comparison of data sets from tracer transport experiments. The comparison can be performed on the basis of parameter estimates since a successful fit implies that the experimental data are fully characterized by the set of model parameters. For this purpose, the absolute values of the fitted model parameters are of little relevance and it is also not necessary, albeit worthwhile, to gain insight into the physiological role of the parameters. Besides, caution has to be taken when trying to interpret a single parameter of the model in a physiological way. This not only includes the exchange and diffusion parameters which sum up several processes within the plant tissues but pertains also to the velocity $v$. Intuitively one might think of $v$ as a group velocity (i.e. the mean velocity of the center of mass), but it denotes the front velocity of the fastest particles (i.e., the particles in compartment 1 ) which might be distinctively different from the group velocity. Therefore, the only parameter with an immediate physiological connotation is the Loss of tracer as a composition of the transport and exchange properties of the system independent of the initial condition $\rho_{0}$. $\rho_{0}$ is a purely auxiliary function used to provide a suitable input into the region of interest. The estimation of the parameters of $\rho_{0}$ during the fitting process is not unlike a backward extrapolation from that region. Therefore, the values of the parameters $\sigma$ and $x_{0}$ of the Gaussian initial distribution used in our examples are of very little interest. The Gaussian distribution
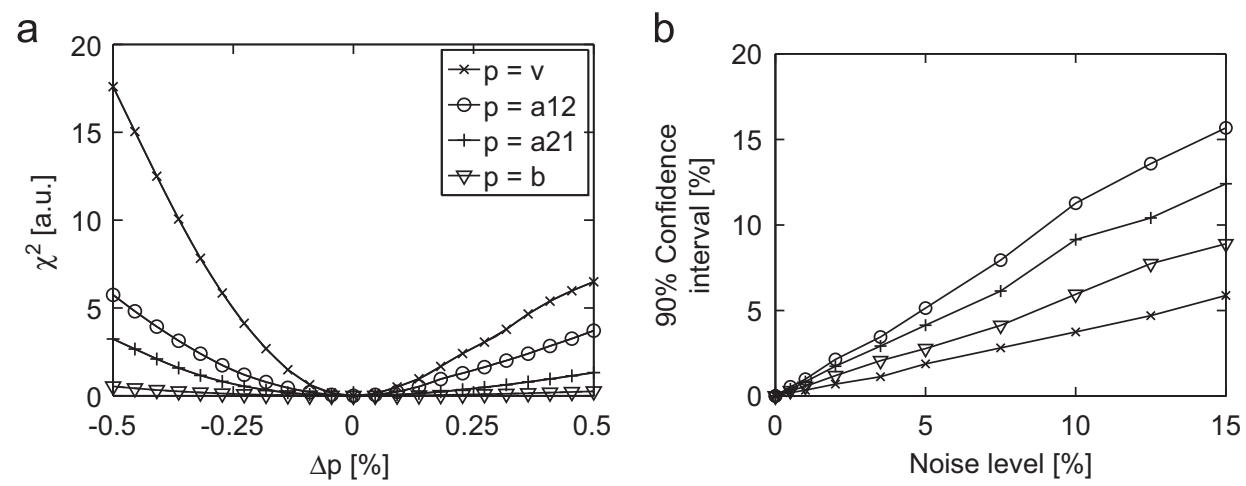

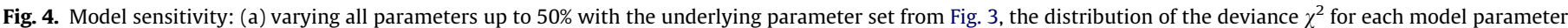
shows the respective sensitivity and (b) confidence intervals of each parameter depending on the level of added noise. 
a

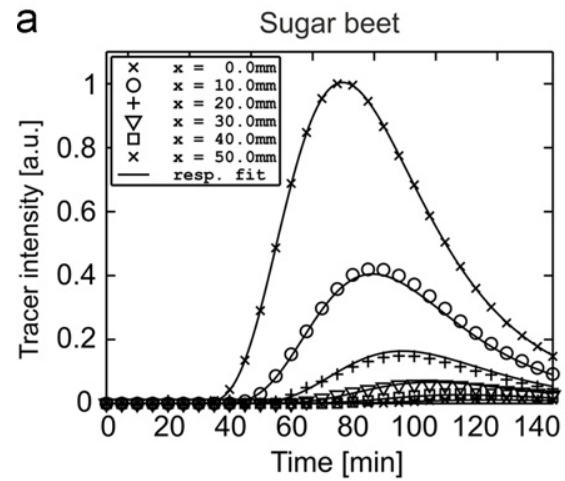

b

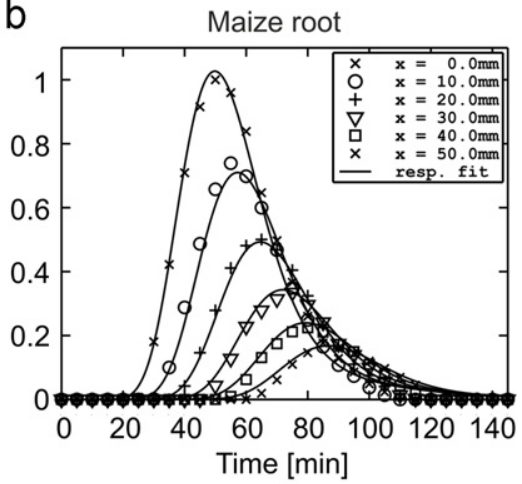

d

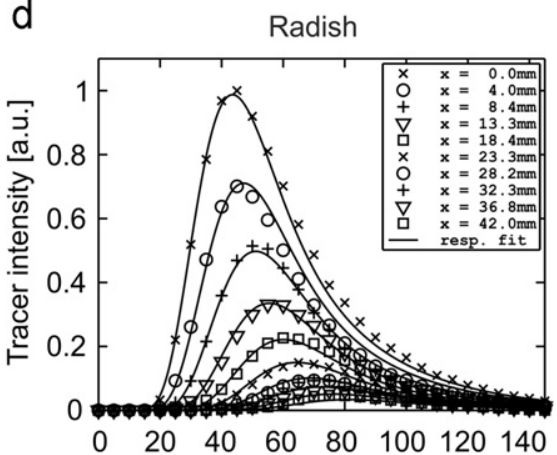

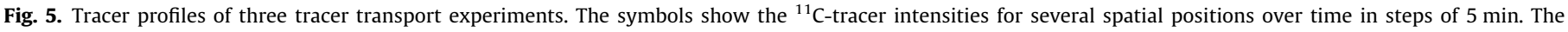

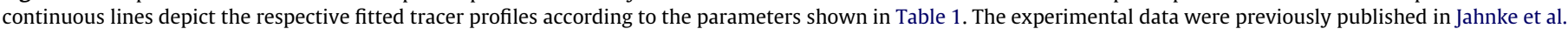

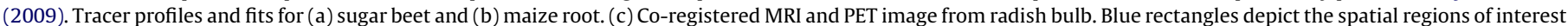
(ROIs) consecutively numbered by symbols on the right side. (d) Tracer profiles from integrated ROIs and fit for radish bulb.

Table 1

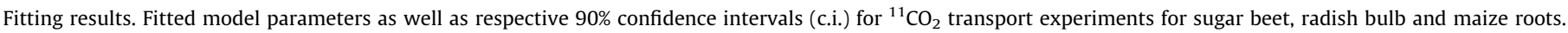
The diffusion rates were not fitted $\left(D_{1,2}=0 \mathrm{~m}^{2} / \mathrm{s}\right), \lambda=0.034 \mathrm{~min}^{-1} \cdot \chi^{2}$ is the deviance. Loss was calculated according to Eq. (5).

\begin{tabular}{|c|c|c|c|c|c|c|c|c|c|c|}
\hline \multirow[t]{2}{*}{ Parameter } & \multirow[t]{2}{*}{ Unit } & \multicolumn{3}{|c|}{ Sugar beet } & \multicolumn{3}{|c|}{ Maize root } & \multicolumn{3}{|c|}{ Radish bulb } \\
\hline & & Fit & & c.i. [\%] & Fit & & c.i. [\%] & Fit & & c.i. [\%] \\
\hline \multicolumn{11}{|l|}{ Model } \\
\hline$v$ & $\mathrm{~mm} / \mathrm{min}$ & 1.99 & \pm & 11.7 & 2.34 & \pm & 34.5 & 2.19 & \pm & 29.5 \\
\hline$a_{12}$ & $\min ^{-1}$ & 0.34 & \pm & 23.1 & 0.32 & \pm & 99.9 & 0.44 & $\overline{ \pm}$ & 67.3 \\
\hline$a_{21}$ & $\min ^{-1}$ & 0.14 & \pm & 10.0 & 0.33 & \pm & 32.0 & 0.24 & \pm & 38.5 \\
\hline$b$ & $\min ^{-1}$ & 0.061 & \pm & 13.1 & 0.016 & \pm & 41.4 & 0.071 & \pm & 23.0 \\
\hline \multicolumn{11}{|c|}{ Initial condition } \\
\hline$\sigma$ & $\mathrm{mm}$ & 6.51 & \pm & 64.0 & 9.84 & \pm & 37.9 & 6.39 & \pm & 54.3 \\
\hline$x_{0}$ & $\mathrm{~mm}$ & 73.33 & \pm & 7.4 & 73.40 & \pm & 4.33 & 44.00 & \pm & 8.7 \\
\hline Loss & $\% / \mathrm{cm}$ & 40.8 & \pm & 3.3 & 6.2 & \pm & 22.9 & 36.4 & \pm & 3.9 \\
\hline$\chi^{2}$ & a.u. & 0.0059 & \pm & 27.8 & 0.0751 & \pm & 19.3 & 0.0477 & \pm & 23.1 \\
\hline
\end{tabular}

was chosen just for convenience; any other smooth initial density function, allowing a similar good fit, could be used instead.

All three examples of experimental data sets are well reproduced by our model, even though the quality of the fits differs as indicated by the value of the deviance $\chi^{2}$. The lower estimate of $b$ and higher ratio of $a_{21}$ to $a_{12}$ for maize (see Table 1 ) results from the lower tail of the experimental curves which is also responsible for the comparably small value of Loss for maize. This was to be expected since in maize roots photoassimilates are transported mainly to the root tips whereas in sugar beet and radish the observed plant parts themselves constitute the storage organs. For all three data sets the confidence intervals of Loss are relatively small which emphasizes the special role of this parameter and its independence from the specific model setup. The confidence intervals of the other model parameter estimates are high compared to what can be explained simply by noise in the data: in our examples the noise is less than $1 \%$ and the analysis of artificial data with added noise in Section 3.3 shows that such a noise level covers only confidence intervals of less than $1 \%$. Thus, the high confidence intervals indicate that for these data the model is over-parameterized although the number of fitting parameters is already small. The over-parameterization can be further demonstrated by the effective parameter reduction obtained through a coupling of two model parameters as explained in Section 3.4. By defining a fixed ratio between parameters $a_{21}$ and $a_{12}$, the confidence intervals reduce considerably depending on the proportionality factor $h$ without 
Table 2

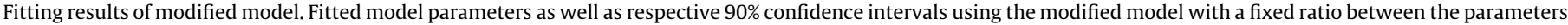
$a_{21}$ and $a_{12}\left(a_{21}=h a_{12}\right) \cdot \chi^{2}$ is the deviance. Loss was calculated according to Eq. (5).

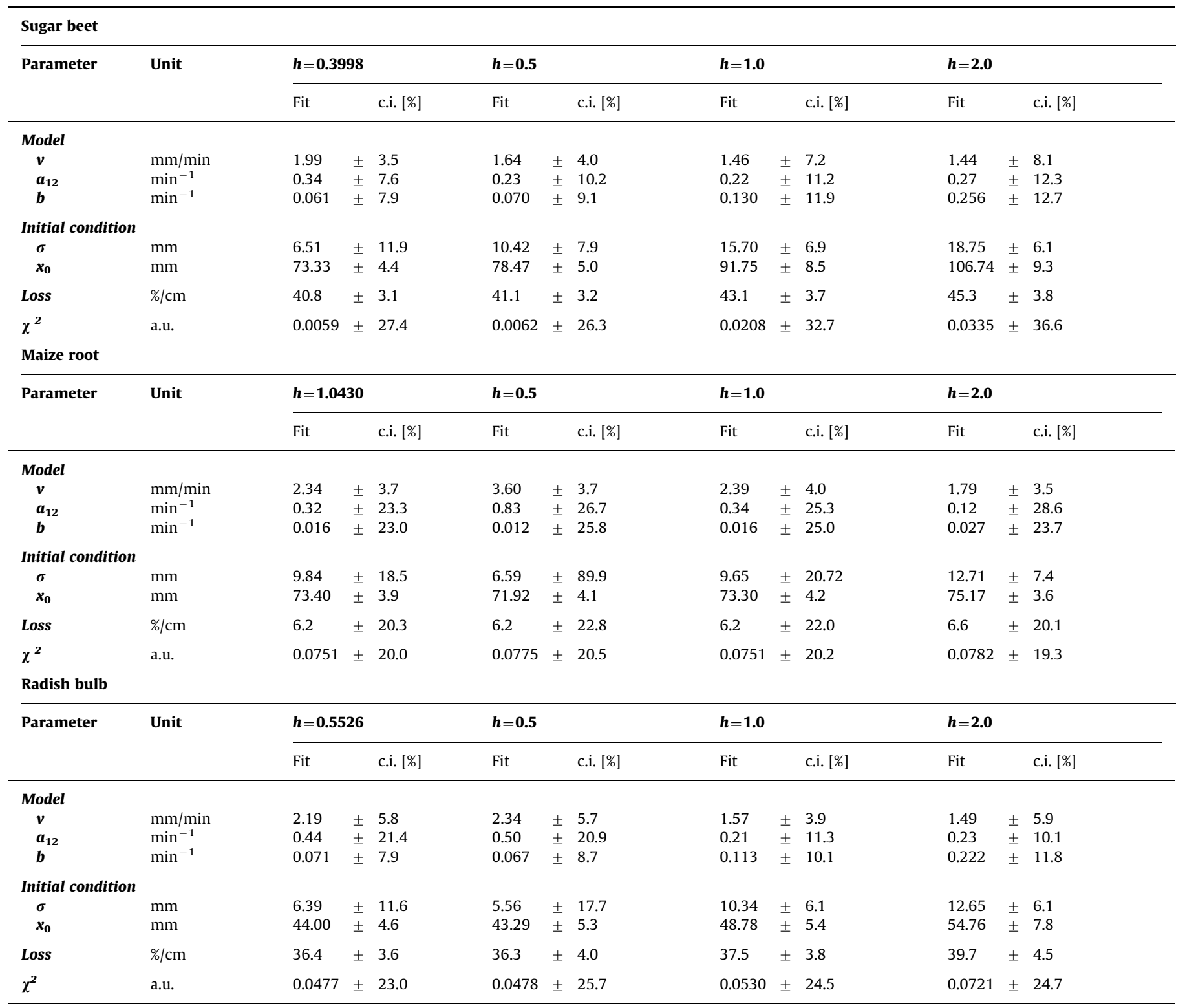

significantly increasing the deviance $\chi^{2}$, see Table 2 . Again, Loss turns out to be stable as it is largely independent of $h$. For the sugar beet and radish data, the $\chi^{2}$ values depend on $h$ while the maize data could always be fitted with the same quality. This indicates that there is no universally optimal ratio of the exchange parameters.

Recognizing the fact of over-parameterization in our examples justifies neglecting additional model parameters like diffusion coefficients. Fitting the model with the diffusion coefficients does not result in a noticeable enhancement of $\chi^{2}$ (the relative decrease is less than $0.1 \%$ ) but in a significant increase of the confidence intervals of all parameters (data not shown). In conclusion, we observe that depending on the informational content of the experimental data, the model needs to be adapted for each specific experimental setup. This can be done by analyzing the co-variances of the parameters and subsequently adding or removing functional parameters in order to accomplish a satisfying parameter estimate.

\section{Conclusions}

Searching for a most simple tracer translocation model describing our experimental data, we extended previously published models (Horwitz, 1958; Tyree, 1975) in several respects: we inserted a third compartment which irreversibly accumulates tracer, included diffusion into the governing equations and obtained an analytical solution for the tracer distribution allowing for fast and accurate calculation. The tracer transport model together with the fitting procedure and error analysis presented constitutes a basic framework for quantitative analysis of data from tracer experiments using nearly arbitrary initial conditions. Although the model parameters are not directly linked to physiological properties, they can be used to calculate the system loss which has an obvious physiological meaning. The example data shown here are well reflected by the model. However, an adaption of the model to specific experimental conditions is still necessary. 
One of the next steps is a systematic investigation of possible covariances of the model parameters and the design of the experimental setup in order to optimize the certainty of the parameters of interest.

\section{Acknowledgments}

We wish to thank Hanno Scharr, Wilfried Wolff, Michael Thorpe and Peter Minchin for helpful discussions. Special thanks go to Siegfried Jahnke for valuable comments and access to the PET data. Jonas Bühler wants to thank Martin Reißel for technical support. Friederike Schmid acknowledges financial support from the MRL of UC Santa Barbara during a sabbatical. This work was partially supported by the MRSEC Program of the National Science Foundation under Award no. DMR05-20415. Finally, Peter Blümler wants to thank Helmut Soltner for an excursion into Laplacian space! Last but not least continuous support from Uli Schurr made this work possible.

\section{Appendix A. Detailed analytical solution}

The system of partial differential equations (1) can be partially compacted using a vector/matrix notation:

$\partial \frac{\rho}{\partial t}=-\left(\underline{\underline{A}}+\underline{\underline{V}} \frac{\partial}{\partial x}-\underline{\underline{D}} \frac{\partial^{2}}{\partial x^{2}}\right) \underline{\rho} \quad$ with

$\stackrel{A}{=}=\left(\begin{array}{cc}a_{12}+\lambda & -a_{21} \\ -a_{12} & a_{21}+b+\lambda\end{array}\right), \underline{\underline{V}}=\left(\begin{array}{ll}v & 0 \\ 0 & 0\end{array}\right)$ and

$\underline{\underline{D}}=\left(\begin{array}{cc}D_{1} & 0 \\ 0 & D_{2}\end{array}\right), \underline{\rho}=\left(\begin{array}{l}\rho_{1}(x, t) \\ \rho_{2}(x, t)\end{array}\right)$

The last equation of (1) only depends on $\rho_{2}(x, t)$ and $\rho_{3}(x, t)$ and will be treated separately. Eq. (A.1) can easily be solved in reciprocal space. The Fourier-transform generates

$\frac{\partial \hat{\rho}(k, t)}{\partial t}=-\left(\underline{A}+i k \underline{\underline{V}}+k^{2} \underline{\underline{D}}\right) \underline{\hat{\rho}}(k, t)$

$\frac{\partial \hat{\rho}_{3}(k, t)}{\partial t}=b \hat{\rho}_{2}(k, t)-\lambda \hat{\rho}_{3}(k, t)$

which has the solution

$\underline{\hat{\rho}}(k, t)=\exp \left[-\left(\underline{\underline{A}}+i k \underline{\underline{V}}+k^{2} \underline{\underline{D}}\right) t\right] \underline{\hat{\rho}}(k, 0)$

$\hat{\rho}_{3}(k, t)=b \int_{0}^{t} \hat{\rho}_{2}(k, \tau) e^{\lambda \tau} d \tau e^{-\lambda t}$

The exponent in Eq. (A.3) can be expressed using the Pauli matrices, $\underline{\underline{\sigma_{j}}}$, as

$\underline{\underline{A}}+i k \underline{\underline{V}}+k^{2} \underline{\underline{D}}=c_{0} \underline{\underline{I}}+\sum_{j=1}^{3} c_{j} \underline{\underline{\sigma_{j}}}$

with $\underline{\underline{I}}=\left(\begin{array}{ll}1 & 0 \\ 0 & 1\end{array}\right), \underline{\underline{\sigma_{1}}}=\left(\begin{array}{ll}0 & 1 \\ 1 & 0\end{array}\right), \underline{\underline{\sigma_{2}}}=\left(\begin{array}{cc}0 & i \\ -i & 0\end{array}\right), \underline{\underline{\sigma_{3}}}=\left(\begin{array}{cc}1 & 0 \\ 0 & -1\end{array}\right)$

which results in the following coefficients:

$c_{0}=\frac{1}{2} \operatorname{Tr}\left(\underline{\underline{A}}+i k \underline{\underline{V}}+k^{2} \underline{\underline{D}}\right)=\frac{1}{2}\left(a_{12}+a_{21}+b+2 \lambda+i k v+k^{2}\left(D_{1}+D_{2}\right)\right)$

$c_{1}=-\frac{1}{2}\left(a_{12}+a_{21}\right), \quad c_{2}=-\frac{1}{2} i\left(a_{12}-a_{21}\right)$

$c_{3}=\frac{1}{2}\left(a_{12}-a_{21}-b+i k v+k^{2}\left(D_{1}-D_{2}\right)\right)$

and the exponential function in Eq. (A.3) becomes

$\exp \left[-\left(\underline{\underline{A}}+i k \underline{\underline{V}}+k^{2} \underline{\underline{D}}\right) t\right]=\exp \left[-c_{0} t\right] \exp \left[-\sum_{j} c_{j} \underline{\underline{\sigma_{j}}} t\right]$
Using the well known commutation and anticommutation relations (see for instance: C. Cohen-Tannoudji, B. Diu, F. Laloë: "Quantum Mechanics" Wiley, 2005)

$\left[\underline{\underline{\sigma_{i}}}, \underline{\underline{\sigma_{j}}}\right]=\underline{\underline{\sigma_{i}}} \underline{\underline{\sigma_{j}}} \underline{\underline{\underline{\sigma_{j}}}} \underline{\underline{\sigma_{i}}}=2 i \sum_{k=1}^{3} \varepsilon_{i j k} \underline{\underline{\sigma_{k}}}$

$\left\{\underline{\underline{\sigma_{i}}}, \underline{\underline{\sigma_{j}}}\right\}=\underline{\underline{\sigma_{i}}} \underline{\underline{\sigma_{j}}}+\underline{\underline{\sigma_{j}}} \underline{\underline{\sigma_{i}}}=2 \delta_{i j} \underline{\underline{I}}$ or $\quad \underline{\underline{\sigma_{i}}} \underline{\underline{\sigma_{j}}}=\delta_{i j} \underline{\underline{I}}+i \sum_{k=1}^{3} \varepsilon_{i j k} \underline{\underline{\sigma_{k}}}$

where $\varepsilon_{i j k}$ is the Levi-Civita symbol and $\delta_{i j}$ is the Kronecker delta, it can be shown that

$$
\left(\sum_{j=1}^{3} c_{j} \underline{=}\right)^{2 n}=|c|^{2 n} \underline{I}=\text { and }\left(\sum_{j=1}^{3} c_{j} \underline{\underline{\sigma_{j}}}\right)^{2 n+1}=|c|^{2 n+1} \sum_{j=1}^{3} \frac{c_{j}}{|c|} \underline{\underline{\sigma_{j}}}
$$

with $|c|=\sqrt{\sum_{j=1}^{3} c_{j}^{2}}$ and $n \in N$

The exponential function in Eq. (A.6) can then be rewritten as

$\exp \left[-\sum_{j} c_{j} \underline{\underline{\sigma_{j}}} t\right]=\cosh \left[\sum_{j} c_{j} \underline{\underline{\sigma_{j}}} t\right]-\sinh \left[\sum_{j} c_{j} \underline{\underline{\sigma_{j}}} t\right]$

where the cosh can be expressed as an expansion of the even powers of the argument while the sinh expands into the odd powers. Combination of Eqs (A.10) with (A.8) then gives

$\exp \left[-\sum_{j} c_{j} \underline{\underline{\sigma_{j}}} \underline{=}\right]=\cosh (|c| t) \underline{\underline{I}}-\sinh (|c| t) \sum_{j=1}^{3} \frac{c_{j}}{|c|} \underline{\underline{\sigma_{j}}}$

This procedure results in the following solution in Fourier-space:

$$
\begin{aligned}
\left(\begin{array}{l}
\hat{\rho}_{1}(k, t) \\
\hat{\rho}_{2}(k, t)
\end{array}\right)= & \exp \left[-c_{0} t\right]\left\{\cosh (|c| t)\left(\begin{array}{ll}
1 & 0 \\
0 & 1
\end{array}\right)\right. \\
& \left.-\frac{\sinh (|c| t)}{|c|}\left(\begin{array}{cc}
c_{3} & c_{1}+i c_{2} \\
c_{1}-i c_{2} & -c_{3}
\end{array}\right)\right\} \underline{\rho}(k, 0)
\end{aligned}
$$

and

$$
\begin{aligned}
\hat{\rho}_{3}(k, t)= & b e^{-\lambda t} \int_{0}^{t} \exp \left[\left(\lambda-c_{0}\right) \tau\right]\left\{\cosh (|c| \tau) \hat{\rho}_{02}(k)\right. \\
& \left.-\frac{\sinh (|c| \tau)}{|c|}\left[\left(c_{1}-i c_{2}\right) \hat{\rho}_{01}(k)-c_{3} \hat{\rho}_{02}(k)\right]\right\} d \tau
\end{aligned}
$$

using $\underline{\hat{\rho}}(k, 0)=\left(\hat{\rho}_{01}(k), \hat{\rho}_{02}(k)\right)^{t}$. Solving the integral in Eq. (A.13) gives

$$
\begin{aligned}
\hat{\rho}_{3}(k, t)= & \frac{b \exp \left(-c_{0} t\right)}{\left(\lambda-c_{0}\right)^{2}-|c|^{2}}\left\{\left[\left(c_{1}-i c_{2}\right) \hat{\rho}_{01}(k)-c_{3} \hat{\rho}_{02}(k)\right]\right. \\
& \times\left[|c| \cosh (|c| t)-\left(\lambda-c_{0}\right) \sinh (|c| t)-|c| \exp \left(\left(c_{0}-\lambda\right) t\right)\right] \\
& -\hat{\rho}_{02}(k)\left[|c| \sinh (|c| t)-\left(\lambda-c_{0}\right) \cosh (|c| t)\right. \\
& \left.\left.+\left(\lambda-c_{0}\right) \exp \left(\left(c_{0}-\lambda\right) t\right)\right]\right\}
\end{aligned}
$$

Inserting Eqs. (A.12) and (A.14) into Eq. (2) with the definitions from Eq. (A.5) gives the final result in a more compact notation avoiding unnecessarily indexed coefficients.

$\hat{\rho}(k, t)=\hat{\rho}_{01}(k) e^{-\lambda t}\left\{e^{-\beta t}\left[\cosh (\delta t)(1-\alpha)+\frac{\sinh (\delta t)}{\delta}\left(a_{12}-\gamma-\alpha \beta\right)\right]+\alpha\right\}$ 


$$
\begin{aligned}
& +\hat{\rho}_{02}(k) e^{-\lambda t}\left\{e ^ { - \beta t } \left[\cosh (\delta t)\left(1-\frac{(\gamma+\beta)}{a_{12}} \alpha\right)\right.\right. \\
& \left.\left.+\frac{\sinh (\delta t)}{\delta}\left(a_{21}+\gamma-\frac{\left(\delta^{2}+\gamma \beta\right)}{a_{12}} \alpha\right)\right]-\frac{(\gamma-\beta)}{a_{12}} \alpha\right\}
\end{aligned}
$$

by using the following definitions:

$$
\begin{aligned}
& \alpha:=\frac{a_{12} b}{\beta^{2}-\delta^{2}} \\
& \beta:=c_{0}-\lambda \\
& \gamma:=c_{3} \\
& \delta:=|c|
\end{aligned}
$$

Eq. (3) is obtained by assuming $\hat{\rho}(k, 0)$ to be non-zero in the first compartment only, i.e. $\hat{\rho}_{0}(k):=\hat{\rho}_{01}(k)$ and $\hat{\rho}_{02}(k)=0$.

\section{Appendix B. Special solution with exchange to compartment 2 only}

Allowing only transport and exchange to the second compartment ( $v>0, a_{12} \geq 0, a_{21}=b=D_{1}=D_{2}=0$ ) and using a Gaussian similar to Eq. (4) as initial condition $\rho_{0}(x)$ the following solution for $\rho_{1}$ and $\rho_{2}$ can be achieved in real space:

$$
\begin{aligned}
\rho_{1}(x, t)= & \frac{1}{\sqrt{2 \pi} \sigma} \exp \left(-\frac{1}{2 \sigma^{2}}\left(x-x_{0}+v t\right)^{2}-\left(a_{12}+\lambda\right) t\right) \\
\rho_{2}(x, t)= & \frac{a_{12}}{2 v} \exp \left\{\frac{a_{12}}{v}\left(x-x_{0}\right)+\frac{a_{12}^{2} \sigma^{2}}{2 v^{2}}-\lambda t\right\} \\
& \times\left[\operatorname{erf}\left(\frac{1}{\sqrt{2}}\left(\frac{x-x_{0}+v t}{\sigma}+\frac{a_{12} \sigma}{v}\right)\right)-\operatorname{erf}\left(\frac{1}{\sqrt{2}}\left(\frac{x-x_{0}}{\sigma}+\frac{a_{12} \sigma}{v}\right)\right)\right]
\end{aligned}
$$

with erf( ) being the Gauss error function. Although not useful for analyzing experimental data, this simple case can be used to give a measure of the numerical error. This error is caused by the discrete Fourier transformation when evaluating Eq. (3) and strongly depends on the choice of the grid for the wave number $k$ (see Section 3.1). The special solution also allows a comparison of the analytical solution equation (3) with a standard PDE solver (e.g. upwind scheme) with regard to convergence (by limiting the grid $k_{\max } \rightarrow \infty$, respectively $\Delta x \rightarrow 0$ for the upwind scheme) and CPUtime consumption, see Appendix C.

\section{Appendix C. Comparison of the analytical solution with a standard PDE solver}

An alternative access to the solution of Eq. (1) can be used for validating the analytical solution. First-order upwind methods (see e.g. Press et al., 2007) are easy to adapt and to implement for our transport model because all model parameters are constant in time and space. For both solutions, the accuracy depends linearly on the spatial grid width $d x$. Decreasing $d x$ (respectively, increasing $k_{\max }$ in case of the analytical solution) results in smaller numerical errors at the cost of higher CPU-time consumptions, see Fig. 6. The numerical error is defined as the maximum deviation from the special solution equation (B.1) using the parameter set $v=1.5 \mathrm{~mm} /$ min, $a_{12}=0.01 \mathrm{~min}^{-1}$ with all other parameters set to zero. The analytical solution shows a linear increase of CPU-time consumption with increasing accuracy whereas the upwind scheme by its nature shows a quadratic dependency. The performance of the upwind scheme does not depend on the number of time points where the model needs to be evaluated as the temporal resolution is always proportional to the spatial grid width $d x$. In contrast, the analytical solution has to be evaluated for each time point which

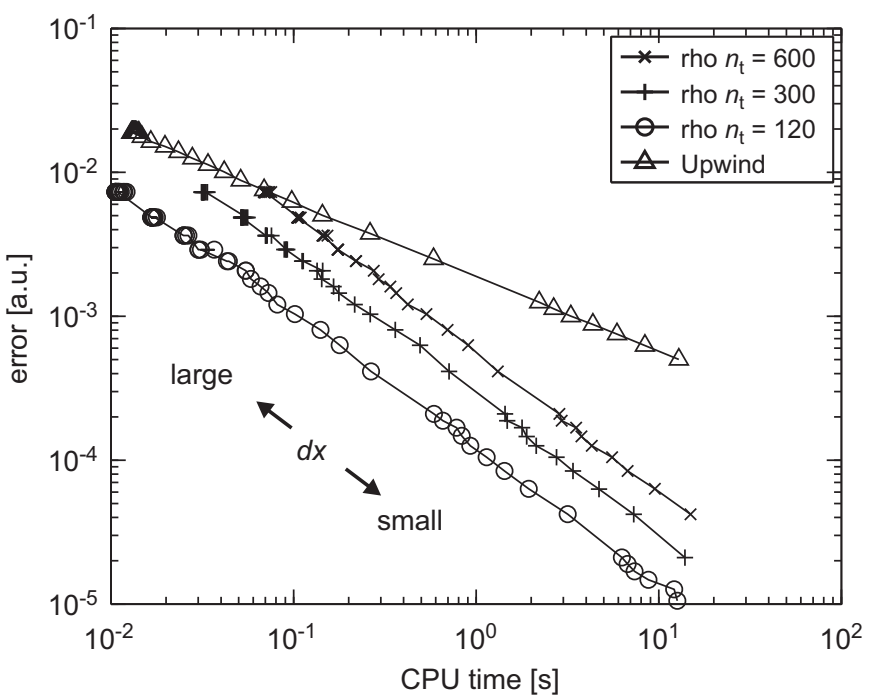

Fig. 6. Comparison with upwind scheme. Comparison of the analytical solution equation (3) and the first-order upwind scheme solving Eq. (1) with regard to the numerical error and the invested CPU-time. The internal grid width $d x$ was varied from 0.001 to $5 \mathrm{~mm}$ for the analytical solution and from 0.04 to $5 \mathrm{~mm}$ for the upwind solution. $n_{t}$ is the number of time points where the model is evaluated.

results in a shift of the error-CPU-time curve for a higher number of time points. For the range of time points considered here the analytical solution is clearly superior to the upwind scheme.

\section{References}

Christy, A.L., Ferrier, J.M., 1973. A mathematical treatment of Munch's pressure-flow hypothesis of phloem translocation. Plant Physiol. 52, 531-538.

De Schepper, V., Steppe, K., 2010. Development and verification of a water and sugar transport model using measured stem diameter variations. J. Exp. Bot. 61 2083-2099.

Goeschl, J.D., Magnuson, C.E., 1986. Physiological implications of the MünchHorwitz theory of phloem transport—effects of loading rates. Plant Cell Environ. 9, 95-102.

Holbrook, N.M., Zwieniecki, M.A., 2005. Vascular Transport in Plants. Elsevier Amsterdam.

Hölttä, T., Vesala, T., Sevanto, S., Peramaki, M., Nikinmaa, E., 2006. Modeling xylem and phloem water flows in trees according to cohesion theory and Munch hypothesis. Trees 20, 67-78.

Horwitz, L., 1958. Some simplified mathematical treatments of translocation in plants. Plant Physiol. 33, 81-93.

Ishida, N., Koizumi, M., Kano, H., 2000. The NMR microscope: a unique and promising tool for plant science. Ann. Bot. 86, 259-278.

Jahnke, S., Menzel, M.I., van Dusschoten, D., Roeb, G.W., Bühler, J., Minwuyelet, S., Blümler, P., Temperton, V.M., Hombach, T., Streun, M., Beer, S., Khodaverdi, M., Ziemons, K., Coenen, H.H., Schurr, U., 2009. Combined MRI-PET dissects dynamic changes in plant structures and functions. Plant J. (59), 634-644.

Köckenberger, W., 2001. Functional imaging of plants by magnetic resonance experiments. Trends Plant Sci. 6, 286-292.

Krantz, W.B., 2007. Scaling Analysis in Modeling Transport and Reaction Processes: A Systematic Approach to Model Building and the Art of Approximation. Wiley New York.

Lacointe, A., Minchin, P.E.H., 2008. Modelling phloem and xylem transport within a complex architecture. Funct. Plant Biol. 35, 772-780.

Legallais, C., Catapano, G., von Harten, B., Baurmeister, U., 2000. A theoretical model to predict the in vitro performance of hemodiafilters. J. Membr. Sci. 168, 3-15.

Levenberg, K., 1944. A method for the solution of certain problems in least squares. Q. Appl. Math. 2, 164-168.

Marquardt, D., 1963. An algorithm for least squares estimation on nonlinear parameters. J. Soc. Ind. Appl. Math. 11, 431-441.

Minchin, P.E.H., Troughton, J.H., 1980. Quantitative interpretation of phloem translocation data. Annu. Rev. Plant Physiol. 31, 191-215.

Minchin, P.E.H., Thorpe, M.R., 2003. Using the short-lived isotope $11 \mathrm{C}$ in mechanistic studies of photosynthate transport. Funct. Plant Biol. 30, 831-841.

Nielsen, H.B., 1999. Damping parameter in Marquardt's method. Available at $\langle$ http://www.imm.dtu.dk/ hbn/publ/TR9905.ps $\rangle$.

Phelps, M.E., Hoffman, E.J., Huang, S.C., Terpogossian, M.M., 1975. Effect of positron range on spatial resolution. J. Nucl. Med. 16, 649-652.

Pickard, W.F., Abraham-Shrauner, B., 2009. A 'simplest' steady-state Münch-like model of phloem translocation, with source and pathway and sink. Funct. Plant Biol. 36, 629-644. 
Press, W.H., Teukolsky, S.A., Vetterling, W.T., Flannery, B.P., 2007. Numerical Recipes: The Art of Scientific Computing. Cambridge University Press, New York.

Risken, H., 1989. The Fokker-Planck Equation. Springer, Berlin.

Thompson, M.V., Holbrook, N.M., 2003. Application of a single-solute non-steadystate phloem model to the study of long-distance assimilate transport. J. Theor. Biol. 220, 419-455.

Thorpe, M.R., Walsh, K.B., Minchin, P.E.H., 1998. Photoassimilate partitioning in nodulated soybean I. 11C methodology. J. Exp. Bot. 49, 1805-1815.

Tyree, M.T., 1975. Horwitz-type models of tracer distribution during unidirectional translocation. In: Aronoff, S. (Ed.), Phloem Transport. Plenum Press, New York, pp. 475-494.
Tyree, M.T., Zimmermann, M.H., 2002. Xylem Structure and the Ascent of Sap. Springer, Heidelberg.

van Deemter, J.J., Zuiderweg, F.J., Klinkenberg, A., 1956. Longitudinal diffusion and resistance to mass transfer as causes of nonideality in chromatography. Chem. Eng. Sci. 5, 271-289.

Vose, P.B., 1980. Introduction to Nuclear Techniques in Agronomy and Plant Biology. Pergamon Press, Oxford.

Windt, C.W., Vergeldt, F.J., De Jager, P.A., Van As, H., 2006. MRI of long-distance water transport: a comparison of the phloem and xylem flow characteristics and dynamics in poplar, castor bean, tomato and tobacco. Plant Cell Environ. 29, 1715-1729. 